\title{
Knockdown of LINC00662 represses AK4 and attenuates radioresistance of oral squamous cell carcinoma
}

\author{
Yangzong Chen, Chunchun Bao, Xiuxing Zhang, Xinshi Lin and Yimou Fu* (i)
}

\begin{abstract}
Background: LncRNAs play crucial roles in the development of carcinomas. However, the investigation of LINC00662 in Oral squamous cell carcinoma (OSCC) is still elusive.

Methods: qRT-PCR assay tested the expression levels of LINC00662, hnRNPC and AK4. With exposure to irradiation, CCK-8, colony formation, flow cytometry and western blot experiments, respectively determined the function of LINC00662 in the radiosensitivity of OSCC cells. Then RIP and western blot assays affirmed the interaction between hnRNPC protein and LINC00662 or AK4. Finally, rescue assays validated the regulation mechanism of LINC00662 in the radioresistance of OSCC.

Results: In the present report, LINC00662 was overexpressed in OSCC and its silencing could alleviate radioresistance of OSCC. Furthermore, the interaction between hnRNPC protein and LINC00662 or AK4 was uncovered. Besides, LINC00662 regulated AK4 mRNA stability through binding to hnRNPC protein. To sum up, LINC00662 modulated the radiosensitivity of OSCC cells via hnRNPC-modulated AK4.
\end{abstract}

Conclusion: The molecular mechanism of the LINC00662/hnRNPC/AK4 axis was elucidated in OSCC, which exhibited a promising therapeutic direction for patients with OSCC.

Keywords: Oral squamous cell carcinoma (OSCC), Radioresistance, LINC00662, hnRNPC, AK4

\section{Background}

Oral squamous cell carcinoma (OSCC) is one of the most aggressive head and neck cancers all over the world [1]. Radiotherapy is a curative therapeutic method for OSCC [2], whereas the effect is still unsatisfactory due to the antergic radioresistance of OSCC [3]. Hence, a better understanding of the molecular regulation mechanism in OSCC was needed.

Long non-coding RNAs (lncRNAs), a sort of non-coding RNAs (ncRNAs), have more than 200 nucleotides in length and play crucial roles in

*Correspondence: YIMOU12321@163.com

Department of Chemotherapy and Radiotherapy, The Second Affiliated Hospital and Yuying Children's Hospital of Wenzhou Medical University, Wenzhou 325027, Zhejiang, China carcinogenesis. Increasing evidence has indicated that aberrantly-expressed lncRNAs participate in cell proliferation, migration, invasion and even the radioresistance of human cancers [4-6]. For example, lncRNA NEAT1 promotes the radio-resistance of cervical cancer by miR-193b-3p/CCND1 axis [7]; lncRNA HOXC13-AS promotes nasopharyngeal carcinoma cell proliferation and invasion via regulating miR383-3p/HMGA2 axis [8]; lncRNA CASC9 positively affects LIN7A expression by miR-758-3p to facilitate ovarian cancer [9]; lncRNA CASC2 downregulation promotes the postoperative local recurrence in early OSCC [10]. Long intergenic non-protein coding RNA 662 (LINC00662), located in chromosome 19, has been reported as an oncogene in colon cancer and hepatocellular carcinoma, influencing the cell growth, cell cycle 
and cell invasion [11, 12]. Besides, LINC00662 boosts cell invasion and cancer stem cell-like phenotypes in lung cancer [13]. LINC00662 promotes OSCC cell proliferation and migration [14]. In this study, we aimed to investigate the role and regulation mechanism of LINC00662 in OSCC cell radioresistance.

RNA-binding proteins usually function as major posttranscriptional regulators by RNA-binding activities [15]. Heterogeneous nuclear ribonucleoprotein C (C1/ $\mathrm{C} 2$ ), also known as hnRNPC, belongs to the subfamily of ubiquitously expressed heterogeneous nuclear ribonucleoproteins (hnRNPs). In past researches, hnRNPC has been illustrated as pivotal mediators in human diseases, including carcinomas $[16,17]$. Besides, hnRNPC is identified as survival-related splicing factor in OSCC [18]. However, the specific functional role and underlying mechanism of hnRNPC in OSCC was uncertain.

Adenylate kinase is a key enzyme in the high-energy phosphoryl transfer reaction in living cells. As an isoform of this enzyme, adenylate kinase 4 (AK4) has been demonstrated as a carcinogen, and plays crucial role in cancer cell resistance to radiation or drugs $[19,20]$. For example, AK4 is a prognostic marker that facilitates metastasis in lung cancer via silencing ATF3, a transcription factor [21]. MiR-199a-3p targets AK4 to influence the multi-chemoresistance of osteosarcoma [19]. MiR-199a-3p targets AK4 to modulate the radioresistance of esophageal cancer cells [20]. The functional role of AK4 has never been investigated in OSCC.

The current study revealed that LINC00662 was highexpressed in OSCC. Mechanism experiments demonstrated that the silencing of LINC00662 improved the radiosensitivity of OSCC cells. Furthermore, hnRNPC protein was uncovered to interact with LINC00662 or AK4. Then, LINC00662 was confirmed to regulate AK4 mRNA stability through binding hnRNPC protein. Totally, upregulated LINC00662 reduced the radiosensitivity of OSCC cells via hnRNPC-modulated AK4 mRNA stability.

\section{Materials and methods \\ Cell culture}

Oral squamous cell carcinoma (CAL27, SCC-4, SCC-1, TU183 and SCC-9) and normal human oral keratinocyte (NHOK) cells were obtained from the cell bank of the Chinese Academy of Sciences (Shanghai, China). Cells were routinely incubated in Dulbecco's modified Eagle medium (DMEM-Hyclone) mixed with 10\% fetal bovine serum (FBS), $100 \mu \mathrm{g} / \mathrm{ml}$ streptomycin and $100 \mathrm{U} / \mathrm{ml}$ penicillin under a $5 \% \mathrm{CO}_{2}$ moist atmosphere at $37^{\circ} \mathrm{C}$.

\section{Cell transfection}

The full-length sequence of hnRNPC or AK4 was subcloned into the pcDNA3.1 plasmid (pcDNA3.1hnRNPC or pcDNA3.1-AK4; Invitrogen,Carlsbad, CA, USA). Knockdown of LINC00662 (sh-LINC00662\#1 or sh-LINC00662\#2) or hnRNPC (sh-hnRNPC) was also achieved by Invitrogen. The transfection was conducted using Lipofectamine 2000 (Invitrogen, Carlsbad, CA, USA) following the instructions of the manufacturer. The sequences of relevant shRNAs are as follows: sh-NC, 5'-GAGCCTGGATGATACATGATGC-3'; shLINC00662\#1， 5'-CTCTTCTATCGAACCGGCCCGC3'; sh-LINC00662\#2, 5'-AGTACTGAACACGGGTTT CAAA-3'; sh-NC, 5'-AGCGAAGGGTCAGGAGAA AGAT-3'; sh-hnRNPC\#1, 5'-CGCTCTCCCCCACAC CCTCTCT-3'; sh-NC, 5'-AAGTGTAAGAGATGAAAG TAGA-3'; sh-AK4\#1， 5'-GCTCTGCGTCTGGTGTGC AACG-3'.

\section{Quantitative real time PCR (qRT-PCR)}

TRIzol (Life Technologies, Carlsbad, CA, USA) was adopted to extract total RNA from OSCC cells and RNA purity was assessed using spectrophotometer (Bio-Rad, Hercules, CA, USA). The Prime-Script miRNA cDNA Synthesis Kit (TaKaRa, Tokyo, Japan) to synthesize cDNA using $1 \mu \mathrm{g}$ of total RNA according to manufacturer's protocol. The SYBR ${ }^{\circledR}$ Premix Ex TaqTM reagent (TaKaRa, Dalian, China) was employed for qPCR analysis on an ABI PRISM 7500 real-time PCR system (Applied Biosystems, Foster City, CA, USA). GADPH was the internal control. The $2^{-\Delta \Delta C T}$ method was adopted to quantify the results. The primer sequences were as follows: LINC00662 forward: 5'-CACGCTTCTGAAACT GGTGT-3', and reverse: 5'-TGTACAGCCTGGTGA CAGAG-3'; AK4 forward: 5'-TGGATTCACCCTCCT AGCGGAA- ${ }^{\prime}$, and reverse: $3^{\prime}$-CTGTCTTAGCCTGGC AGCAACT-5'; hnRNPC forward: 5'-TGGGCTGCTCTG TTCATAAGGG-3', and reverse: $5^{\prime}$-CTCGGTTCACTT TTGGCTCTGC-3'; GAPDH forward: 5'-GCACCGTCA AGGCTGAGAAC-3', and reverse: 5'-TGGTGAAGA CGCCAGTGGA-3'.

\section{Cell counting kit-8 (CCK-8) assay}

CCK-8 assay measured the proliferation capacity of cells. Transfected cells were seeded into 96-well culture dishes with a density of $3 \times 10^{3}$ cells/well for 12 -h culture with or without 4Gy radiation treatment at the dose rate of $200 \mathrm{cGy} / \mathrm{min}$ using ${ }^{137} \mathrm{Cs} \gamma$-ray source (Atomic Energy of Canada Ltd, Mississauga, Ontario, Canada). Then, cell counting kit-8 (10 $\mathrm{\mu l} /$ well, Dojindo, Kumamoto, Japan) was added according to manufacturer's protocol, 
followed by incubation at $37^{\circ} \mathrm{C}$ for another $3 \mathrm{~h}$. Absorbance at $450 \mathrm{~nm}$ was detected by the MRX II microplate reader (Dynex, Chantilly, VA, USA).

\section{Colony formation assay}

Transfected OSCC cells were inoculated into a 6-well culture dish (500 cells/well) and were exposed to several radiation doses ( $0,2,4$ and $8 G y)$. All cells were cultured for 2 weeks, then washed by PBS, fixed by $10 \%$ formaldehyde, and stained by $0.1 \%$ Crystal Violet (Sigma, U.S.A.). The number of colonies more than 50 cells was counted, based on which cell survival fraction (plating efficiency $(\mathrm{PE}) ; \mathrm{SF}=$ each dose of $\mathrm{PE} /$ non-irradiated $\mathrm{PE} \times 100 \%$ ) was calculated.

\section{Flow cytometry of cell apoptosis and cell cycle}

For cell apoptosis analysis, cells were inoculated into six-well plates and transfected with sh-NC or shLINC00662\#2 for $48 \mathrm{~h}$, and then treated with a 4Gy irradiation for $24 \mathrm{~h}$. OSCC cells were washed twice by PBS, centrifuged to isolate the debris and then re-suspended in binding buffer at a concentration of $1 \times 10^{6}$ cells per ml. Cells $\left(1 \times 10^{6}\right)$. Afterwards, cells were dyed with Annexin $\mathrm{V} /$ propidium iodide (PI) reagents (KeyGen Biotech, Nanjing, China) according to the instructions of the manufacturers. After the addition of binding buffer $(0.4 \mathrm{ml})$, cells were examined by a BD FACSCanto II flow cytometer (BD Biosciences, San Jose, CA, USA). As for cell cycle analysis, cells were co-cultured in 6 -well plates $\left(3 \times 10^{5}\right.$ cells/well) with propidium iodide (PI) staining buffer (Dojindo Molecular Technologies, Inc) and subjected to flow cytometer (BD Biosciences). The proportion of cells in G0/G1, S, or G2/M phases was analyzed with the Cell Quest Pro acquisition software (BD Biosciences).

\section{Flow cytometry of transfection efficiency}

The indicated cells $\left(5 \times 10^{5}\right)$ were seeded into each well of 24-well plates and cultured with complete DMEM for $24 \mathrm{~h}$ prior to transfection. The cells treated alone with pSEB pooled plasmids containing transfection plasmids were named as group I; with pSEB plasmids with ultrasound were named as group II; with the lipid microbubble loaded with transfection plasmids were named as group III; with ultrasound and the lipid microbubble loaded with transfection plasmids were named as group IV; with non-plasmid control were named as group V. The Lipofection group (Lipo) was used for comparing transfection efficiency. Cells of group II and IV were ultra-sonicated with $1 \mathrm{MHz}$ of radiation frequency, pulse wave and $0.5 \mathrm{~W} / \mathrm{cm}^{2}$ of sound intensity for $30 \mathrm{~s}$. After that, the transfected cells were harvested after $48 \mathrm{~h}$ for flow cytometry to detect transfection efficiency.

\section{Transwell assays}

Cell migratory ability was evaluated with the use of the Transwell polycarbonate membrane inserts (Millipore, Billerica, MA, USA). The transfected CAL27 and SCC-4 cells $\left(1 \times 10^{5}\right)$ were planted into the upper chamber of the insert. 10\% FBS was used to supplement cell-free medium in the low chamber at $37^{\circ} \mathrm{C}$ for 1 day. Cells migrating through the membranes were cultured in $4 \%$ paraformaldehyde and stained with $0.1 \%$ crystal violet. For cell invasion assay, $1 \times 10^{5}$ cells in sterile medium were seeded into the upper chamber with Matrigel (Sigma-Aldrich, St. Louis, MO, USA). Both migrated and invaded cells were observed and counted under an inverted light microscope (Leica Microsystems, Wetzlar, Germany) at a magnification of $\times 200$.

\section{Knockout of LINC00662 or hnRNPC by CRISPR/Cas9}

The CRISPR/Cas9 genome editing system was applied for generating the LINC00662 or hnRNPC knockout cells. The two sgRNAs targeting LINC00662 or hnRNPC were individually transfected into cells and Cas9-EGFP vector. The pCRISPR-LvSG03 for sgRNAs and CP-LvC9NU-02 for construction of Cas9-EGFP were all procured from Genecopoeia (Rockville, MD, USA). Cas9-expressing cells were established before co-infection of two sgRNAs. The genomic deletions of isolated single colonies were detected using T7 endonuclease I cleavage and PCR, and determined with sequencing after cloning the relative PCR products to $\mathrm{T}$ vector (Takara).

\section{Bioinformatics prediction}

starBase v2.0 (http://starbase.sysu.edu.cn/starbase2/ browseClipSeq.php) was employed to predict the RNAbinding proteins of LINC00662 or AK4. In the ProteinRNA column, we chose protein-lncRNA interactions and protein-mRNA interactions parts, respectively. Among the putative LINC00662-binding proteins and AK4binding proteins, we selected hnRNPC, one of the top ten RNA-binding protein candidates according to starBase v3.0 (http://starbase.sysu.edu.cn/), for further studies.

\section{RNA immunoprecipitation (RIP) assay}

For RNA immunoprecipitation (RIP) assay, a Magna RIP Kit (Millipore, Billerica, MA, USA) was adopted according to the manufacturer's protocols. The whole cell lysate was incubated in RIP buffer where magnetic beads were absorbed by anti-hnPNRC (ab133607; 1:100; Abcam, Cambridge, MA, USA) or anti-IgG (ab218427; 1:100; CST, Boston, MA, USA). IgG was a normalization control. After the digestion of proteinase K, co-precipitated 
RNAs including LINC00662 and AK4 were harvested, purified and detected by qRT-PCR.

\section{RNA pull-down assay}

LINC00662 or AK4 sense was named as LINC00662-WT or AK4-WT, compared with LINC00662 or AK4 antisense (LINC00662-Mut or AK4-Mut). LINC00662-WT, LINC00662-Mut, AK4-WT and AK4-Mut were in vitro transcribed by TranscriptAid T7 High Yield Transcription Kit (Thermo Fisher Scientific, Inc., Waltham, MA, USA) and subsequently biotin-labelled through the Biotin RNA Labelling Mix (Roche) and T7 RNA polymerase (Roche) as instructed. After the incubation with DNase I (Takara), the RNeasy Mini Kit (Qiagen, Valencia, CA, USA) was utilized to purify the transcribed RNAs. Then, $3 \mu \mathrm{g}$ of biotinylated RNAs were grown for $1 \mathrm{~h}$ at $25^{\circ} \mathrm{C}$ in mixture with $1 \mathrm{mg}$ whole cell lysates. Then, the complexes were extracted via streptavidin-conjugated Dynabeads (Invitrogen). Eventually, the pull-down materials were confirmed by western blot.

\section{Western blot}

CAL27 and SCC-4 cells were cultured in RIPA buffer with protease inhibitor cocktails (AMRESCO), lysed for $30 \mathrm{~min}$ with brief vortexing every $10 \mathrm{~min}$. Then centrifuge the lysates for $15 \mathrm{~min}$ at $4{ }^{\circ} \mathrm{C}$ and detect protein concentrations using the BCA Protein Assay Reagent (Pierce, Appleton, Wisconsin, USA). Post separation of $40 \mu \mathrm{g}$ protein by sodium dodecyl sulfate polyacrylamide gel electrophoresis (SDS-PAGE), the lysate was transferred to nitrocellulose membranes. The membranes were incubated with primary antibodies against cleaved PARP (ab32064; Abcam), PARP (ab74290; Abcam), cleaved caspase-3 (ab2302; Abcam), caspase-3 (ab13847; Abcam), hnRNPC (ab97541; Abcam), AK4 (ab232888; Abcam) or GAPDH (sc-32233; Santa Cruz Biotechnology, Santa Cruz, CA, USA) at $4{ }^{\circ} \mathrm{C}$ overnight. After washing, the membrane was cultured with horseradish peroxidaseconjugated (HRP) secondary antibody (Santa Cruz Biotechnology, Inc., Dallas, TX, USA) at room temperature for $2 \mathrm{~h}$. Finally, the signals were estimated by enhanced fluorescence as suggested.

\section{Statistical analysis}

All statistical analyses were conducted via SPSS 18.0 (SPSS, Inc., Chicago, IL, USA) and GraphPad Prism software (GraphPad, Inc., La Jolla, CA, USA). The data were indicated as the mean \pm standard deviation. Statistical significance analysis of two or multiple groups was performed using Student's t-test or ANOVA. P value less than 0.05 was accepted as statistically significant. Each assay was independently carried out in triplicate (Additional file 1).

\section{Results LINC00662 silencing attenuated radioresistance of OSCC cells}

To explore the expression profiles of LINC00662 in OSCC, we firstly tested its expression in OSCC cell lines (CAL27, SCC-4, SCC-1, TU183 and SCC-9) and the primary normal human oral keratinocytes (NHOK) cell lines. qRT-PCR revealed that the expression of LINC00662 was relatively up-regulated in OSCC cells (Fig. 1a). The sensitivity of SCC-4 and CAL27 cells to radiation was significantly lower than that of normal NHOK cell (Additional file 2: Figure S1A). Among the incubated OSCC cell lines, CAL27 and SCC-4 cells with the highest level of LINC00662 were chosen for subsequent experiments. LINC00662 expression was further enhanced in CAL27 and SCC-4 cells under 4Gy irradiation at a time dependent manner, compared to no irradiation exposure (Fig. 1b). In order to investigate the effect of LINC00662 on the radioresistance of OSCC, shLINC00662\#1 or sh-LINC00662\#2 was transfected into CAL27 and SCC-4 cells. LINC00662 expression was significantly reduced by transfection of sh-LINC00662\#1/2, as examined by qRT-PCR (Fig. 1c and Additional file 1A). Here, since sh-LINC00662\#2 exhibited higher transfection efficiency than sh-LINC00662\#1, the knockdown effect of sh-LINC00662\#2 was better than that of shLINC00662\#1, and we selected sh-LINC00662\#2 for later assays. CCK-8 assay illustrated that $4 \mathrm{~Gy}$ irradiation or LINC00662 repression inhibited cell proliferation of CAL27 and SCC-4 cells, and this effect was strengthened under the co-treatment of 4Gy irradiation and LINC00662 knockdown (Fig. 1d). Colony formation assay

\footnotetext{
(See figure on next page.)

Fig. 1 LINC00662 attenuated radioresistance of OSCC cells. a qRT-PCR analysis of LINC00662 expression in OSCC (CAL27, SCC-4, SCC-1, TU183 and SCC-9) and normal human oral keratinocytes (NHOK) cell lines. b LINC00662 expression in CAL27 and SCC-4 cells under 4Gy irradiation was tested by qRT-PCR. c The expression of LINC00662 by transfection of sh-LINC00662\#1 or sh-LINC00662\#2 in CAL27 and SCC-4 cells was also measured by qRT-PCR. $\mathbf{d}$ CCK-8 assay was performed to examine cell viability of sh-LINC00662\#2 transfected CAL27 and SCC-4 cells under 0 or 4 Gy radiation, compared with relative control groups. e Survival fractions of sh-LINC00662\#2 treated CAL27 and SCC-4 cells at the indicated doses of 0, 2, 4 and $8 \mathrm{~Gy}$ radiation were respectively determined by colony formation assay. $\mathbf{f}$ Flow cytometry analysis of cell apoptosis in CAL27 and SCC-4 cells with LINC00662 down-regulation after 0 or 4Gy irradiation treatment. $\mathbf{g}$ Under 0 or 4Gy irradiation, cleaved PARP, cleaved caspase-3, total PARP and caspase-3 levels in CAL27 and SCC-4 cells with LINC00662 silencing were detected through western blot. ${ }^{*} P<0.05$, **P $<0.01$, and ${ }^{* * * P}<0.001$
} 


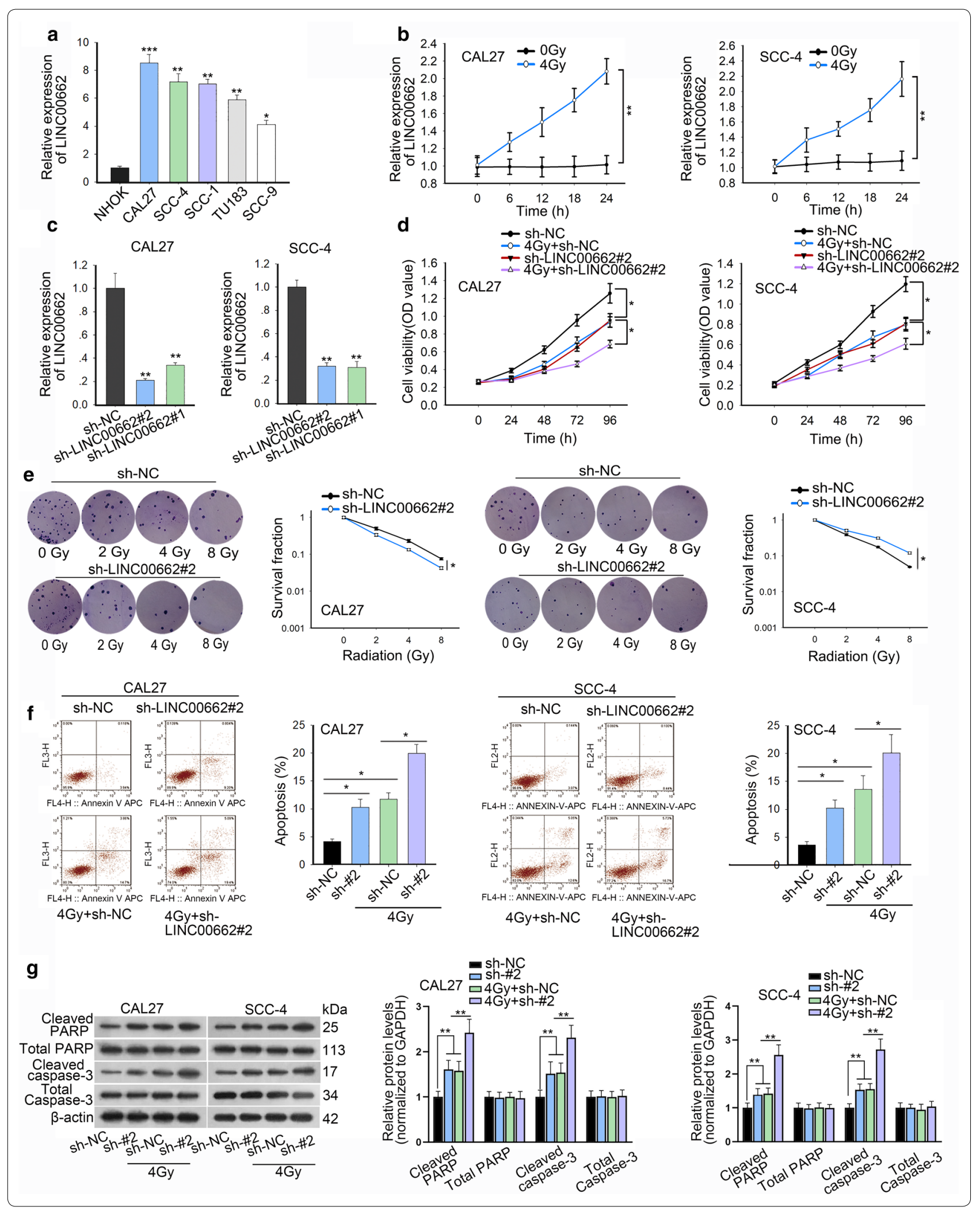


a

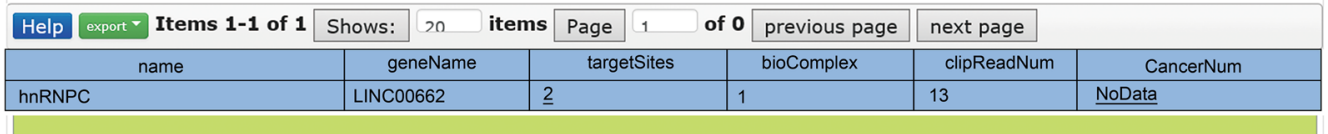

b

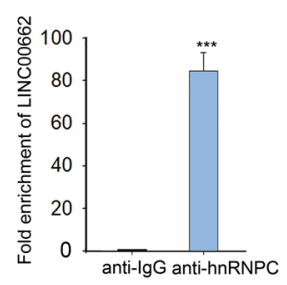

c
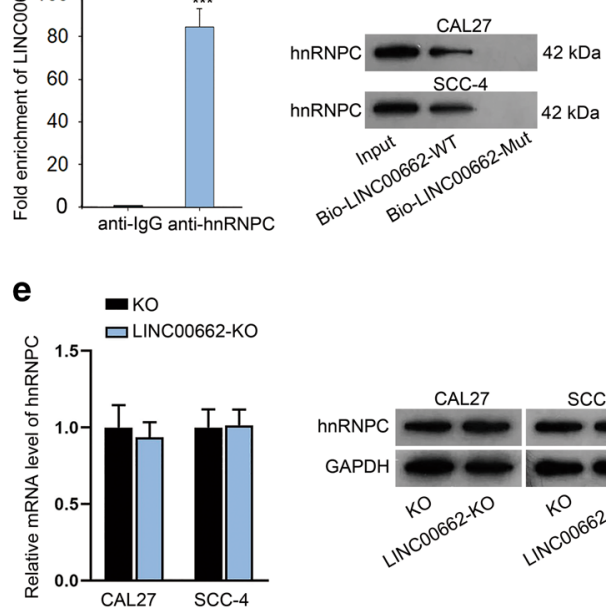

\section{d}
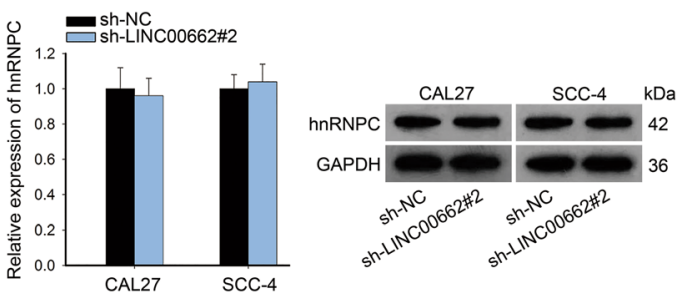

Fig. 2 LINC00662 bound with hnRNPC protein and had no regulatory influence on hnRNPC. a The predicted binding between LINC00662 and hnRNPC was obtained from starBase. b-c RIP and western blot assays validated the interaction of LINC00662 and hnRNPC protein. $\mathbf{d}$ hnRNPC expression had no alteration when LINC00662 was knocked down, as estimated by qRT-PCR and western blot. e qRT-PCR and western blot revealed influence of LINC00662 knockout on hnRNPC expression. ${ }^{* *} \mathrm{P}<0.001$

indicated that there was a declining trend of cell survival fraction with the increased irradiation doses. LINC00662 down-regulation could also restrain survival fraction in both CAL27 and SCC-4 cells. Importantly, combination of irradiation and LINC00662 silencing markedly reinforced the declining trend of cell survival fraction (Fig. 1e). Flow cytometry cell cycle analysis showed that radiation or LINC00662 silence arrested cell cycle at G0/ G1 phrase. Silenced LINC00662 further increased proportion of cells at G0/G1 phrase caused by 4Gy radiation (Additional file 2: Figure S1B). In flow cytometry assay, apoptosis was obviously enhanced by radiation exposure or by transfection of sh-LINC00662\#2. Combination of radiation exposure and the knockdown of LINC00662 further enhanced cell apoptosis rates of CAL27 and SCC-4 cells (Fig. 1f). Poly ADP-ribose polymerase (PARP) is a kind of DNA repairing enzyme and also an apoptosis-related protein [22]. Western blotting demonstrated that the expression level of cleaved-PARP and cleaved caspase-3 decreased after 4Gy irradiation or silence of LINC00662. Such effect was further significantly boosted through the coexistence of radiation and LINC00662 inhibition. In the meanwhile, expression of total PARP or caspase-3 showed no changes to 4Gy irradiation or silence of LINC00662 (Fig. 1g). Besides, cell motility was also assessed via transwell assays. The results exhibited that LINC00662 silencing further aggravated the suppressive effects of $4 \mathrm{~Gy}$ irradiation on cell migration and invasion (Additional file 2: Figure S1C and D).

Moreover, we constructed the LINC00662-KO plasmid to knock out LINC00662 expression. LINC00662 expression was significantly knocked out by transfection of LINC00662-KO (Additional file 3: Figure S2A). All the functional assays demonstrated that LINC00662-KO significantly enhanced OSCC cell sensitivity to radiation exposure (Additional file 3: Figure $\mathrm{S} 2 \mathrm{~B}-\mathrm{H}$ ).

In conclusion, all these data revealed that LINC00662 served as an oncogene in OSCC cells, and LINC00662 knockdown or knockout improved radiosensitivity of OSCC cells.

\section{LINC00662 bound with hnRNPC protein and had no regulatory influence on $h n R N P C$}

Past researches have unveiled that RNA-binding proteins (RBPs) can interact with lncRNAs in tumors [23]. From starBase, we discovered that LINC00662 potentially bound with hnRNPC protein (Fig. 2a). RIP and western blot assays were performed to confirm the combination of LINC00662 with hnRNPC protein. In RIP experiment, the mixtures immunoprecipitated by anti-hnRNPC 
exhibited an obvious enrichment of LINC00662 (Fig. 2b). The western blot assay demonstrated that LINC00662 interacted with hnRNPC protein (Fig. 2c). Moreover, the mRNA and protein level of hnRNPC exhibited no change in response to sh-LINC00662\#2 group, compared with control group (Fig. 2d). Besides, knockout of LINC00662 had no influences on the mRNA and protein level of hnRNPC (Fig. 2e). These results proved that LINC00662 bound to hnRNPC protein and had no regulatory influence on hnRNPC.

\section{hnRNPC bound with AK4 mRNA to stabilize AK4 mRNA}

RNA-binding proteins are also reported to interact with mRNAs and further stabilize their mRNA expression levels [24]. From starBase, we found the potential combination of AK4 with hnRNPC (Fig. 3a). As AK4 was reported to modulate the radioresistance of cancer cells [20], we guessed that AK4 might participate in LINC00662mediated radioresistance in OSCC cells. RIP assay illustrated that the compounds precipitated by anti-hnRNPC exhibited abundant enrichment of AK4 (Fig. 3b). RNA pull-down and western blot assays further verified that hnRNPC protein bind with bio-AK4-WT (Fig. 3c). Furthermore, we probed the effects of hnRNPC on AK4. The expression level of hnRNPC was markedly knocked down at both mRNA and protein level after sh-hnRNPC was stably transfected into CAL27 and SCC-4 cells (Fig. 3d and Additional file 1B). As a result, AK4 expression was significantly reduced by knockdown of hnRNPC (Fig. 3e), as measured by qRT-PCR. Besides, qRT-PCR displayed that the remaining mRNA of AK4 was reduced when actinomycin $\mathrm{D}$ was added, and this phenomenon was aggravated by the knockdown of hnRNPC, showing the positive regulation of hnRNPC on the stability of AK4 mRNA (Fig. 3f). After that, we constructed hnRNPC-KO plasmids to evaluate the effects of hnRNPC knockout on AK4. hnRNPC expression was significantly knocked out at both mRNA and protein level after stable transfection of hnRNPC-KO (Fig. 3g). Knockout out of hnRNPC remarkably reduced AK4 expression (Fig. 3h). Also, knockout out of hnRNPC aggravated degradation of AK4 caused by actinomycin D (Fig. 3i). Collectively, hnRNPC protein interacted with AK4 mRNA and further stabilized AK4 mRNA.

\section{LINC00662 promoted AK4 mRNA stability through binding to hnRNPC protein}

LncRNAs have been revealed to interact with RBPs and then regulate the stability of their target genes [25]. Hence, we supposed that LINC00662 modulated AK4 mRNA stability via binding to hnRNPC protein. qRTPCR and western blot analysis revealed the up-regulated expression of AK4 in OSCC cell lines, in comparison with
NHOK cell lines (Fig. 4a). By qRT-PCR and western blot analysis, AK4 expression was overtly downregulated at both mRNA and protein levels on account of LINC00662 inhibition (Fig. 4b, c). Also, knockout of LINC00662 dramatically reduced AK4 expression at both mRNA and protein level (Fig. 4d). Then, we detected the influence of LINC00662 overexpression on AK4 expression. Transfection of pcDNA3.1-LINC00662 significantly enhanced expression of AK4 at both mRNA and protein level (Fig. $4 \mathrm{e}-\mathrm{f}$ and Additional file 1C) Thereafter, pcDNA3.1hnRNPC was transfected into CAL27 and SCC-4 cells. Transfection of pcDNA3.1-hnRNPC resulted in the overexpression of hnRNPC, as determined by qRT-PCR and western blot (Fig. 4g and Additional file 1D). Finally, qRT-PCR demonstrated that in both CAL27 and SCC-4 cells, actinomycin D inhibited the RNA synthesis of AK4, which was exacerbated after LIN00662 was downregulated but restored when hnRNPC was upregulated (Fig. 4h). Furthermore, RIP experiment was performed to explore whether LINC00662 served as a scaffold for the binding between hnRNPC and AK4. The results displayed that the binding of hnRNPC with AK4 was blocked by sh-LINC00662\#2 but recovered by pcDNA3.1-hnRNPC (Fig. 4i). Taken together, we concluded that LINC00662 recruited hnRNPC protein to increase AK4 expression.

\section{LINC00662 modulated the radiosensitivity of OSCC cells via AK4}

Rescue assays were performed to affirm the function of LINC00662/hnRNPC/AK4 axis in OSCC. We firstly evaluated function of AK4 overexpression in OSCC cell radioresistance. In qRT-PCR assay, AK4 expression was significantly up-regulated at approximately 8.5fold increase in CAL27 cells and approximately 6.2-fold increase in SCC-4 cells by transfection of pcDNA3.1AK4; the overexpression efficiency of AK4 was also verified in the following western blot assay (Fig. 5a and Additional file 1E). The gain of function assays depicted that AK4 overexpression enhanced cell radioresistance by promoting cell viability (Fig. $5 \mathrm{~b}$ ), proliferation (Fig. 5c), inhibiting cell cycle arrest (Fig. 5d) and apoptosis (Fig. 5f), facilitating migration (Fig. $5 \mathrm{~g}$ ) and invasion (Fig. 5h).

Next, the rescue assays were conducted using AK4 overexpression to rescue LINC00662 knockdown in cell radio-resistance. CCK-8 experiment demonstrated that under 4Gy irradiation, cell viability of CAL27 and SCC-4 cells was further repressed by LINC00662 knockdown but rescued by AK4 overexpression (Fig. 6a). In colony formation assay, the increasing of radiation decreased the survival fraction of CAL27 and SCC-4 cells gradually, which was reinforced by the silencing of LINC00662 but restored by the overexpression of AK4 (Fig. 6b). Under 
a

\begin{tabular}{|c|c|c|c|c|c|c|c|c|}
\hline Help export Items 1-1 of 1 & Shows: & 20 & items & Page & 1 & of 0 & previous page & next page \\
\hline
\end{tabular}

\begin{tabular}{|l|l|l|l|l|l|l|}
\hline \multicolumn{1}{|c|}{ name } & \multicolumn{2}{c|}{ geneName } & targetSites & bioComplex & clipReadNum & CancerNum \\
\hline hnRNPC & AK4 & $\underline{6}$ & 1 & 61 & NoData \\
\hline
\end{tabular}

b
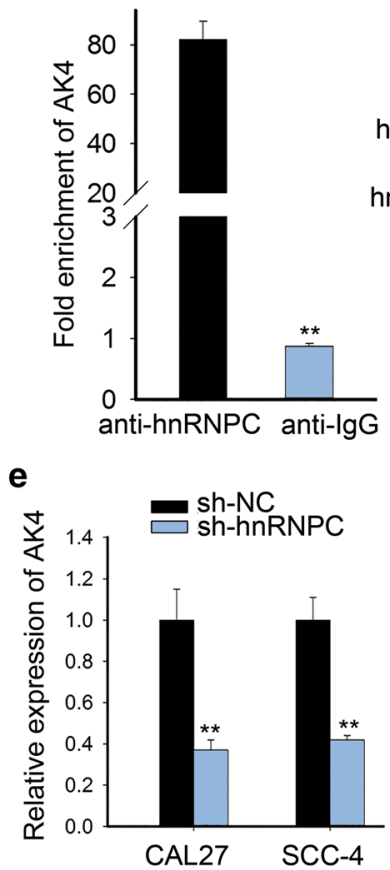

$\mathbf{h}$

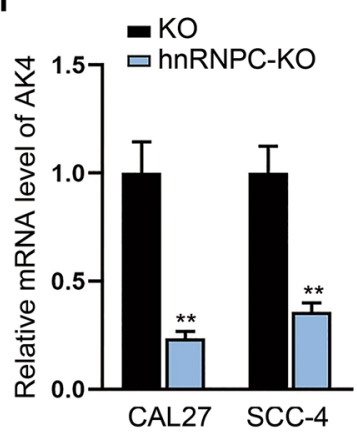

C

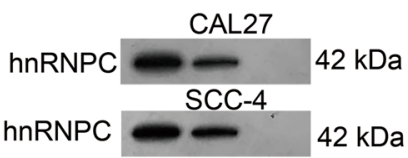

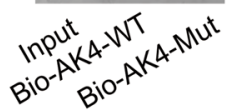
$42 \mathrm{kDa}$

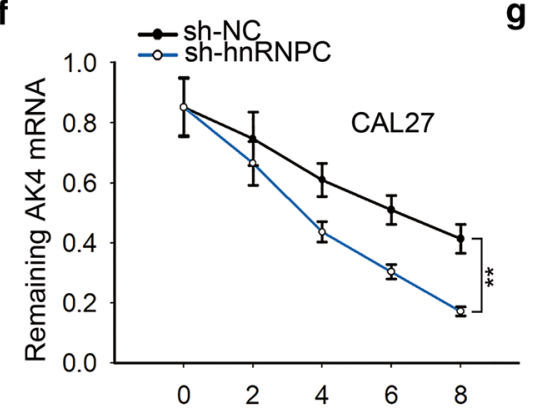

Hours post Actinomycin D

i

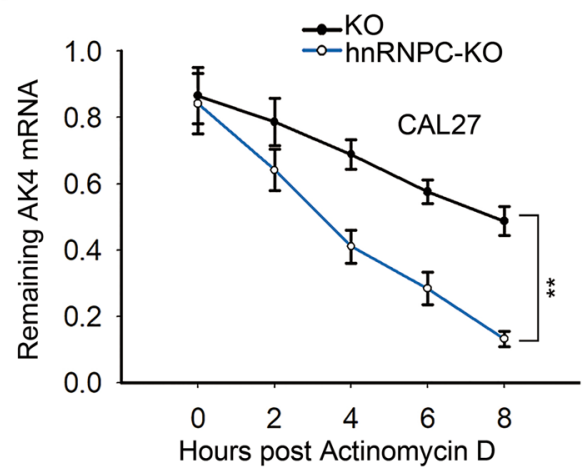

Fig. 3 hnRNPC bound with AK4 mRNA to stabilize AK4 mRNA. a The putative binding capacity between hnRNPC and AK4 was predicted from starBase. $\mathbf{b}, \mathbf{c}$ The combination of hnRNPC with AK4 was confirmed by RIP and western blot assays. $\mathbf{d}$ qRT-PCR and western blot evaluated hnRNPC expression in sh-hnRNPC transfected CAL27 and SCC-4 cells. e qRT-PCR detection of AK4 level in CAL27 and SCC-4 cells with the transfection of sh-hnRNPC. $\mathbf{f}$ Under actinomycin D treatment, the mRNA stability of AK4 was assessed through qRT-PCR by knockdown of hnRNPC. $\mathbf{g}$ qRT-PCR and western blot evaluated hnRNPC expression in hnRNPC-KO transfected CAL27 and SCC-4 cells. $\mathbf{h}$ qRT-PCR revealed AK4 expression by knockout of hnRNPC. $\mathbf{i}$ Under actinomycin D treatment, the mRNA stability of AK4 was assessed through qRT-PCR by knockout of hnRNPC. ${ }^{* * P}<0.01$ 


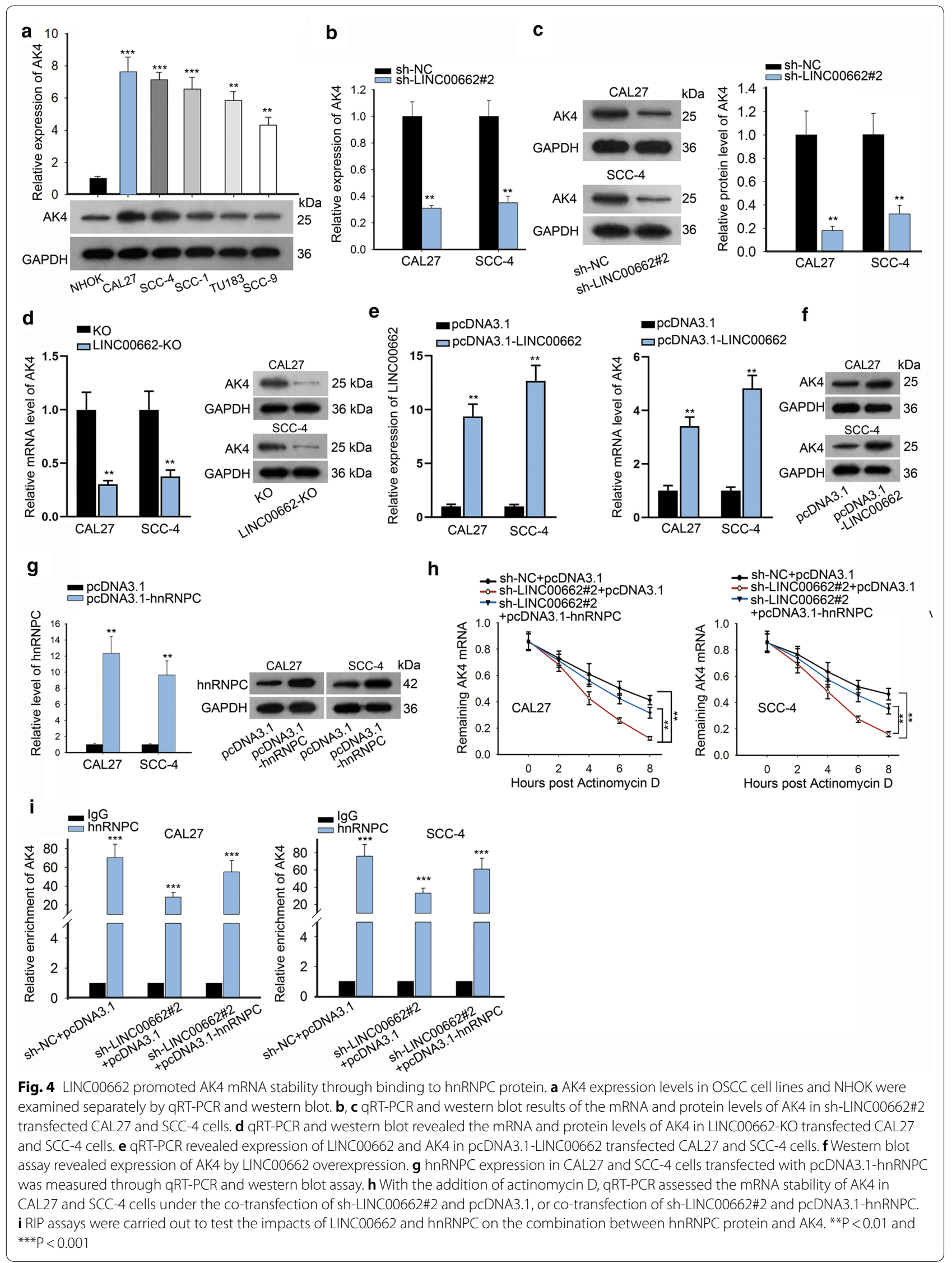




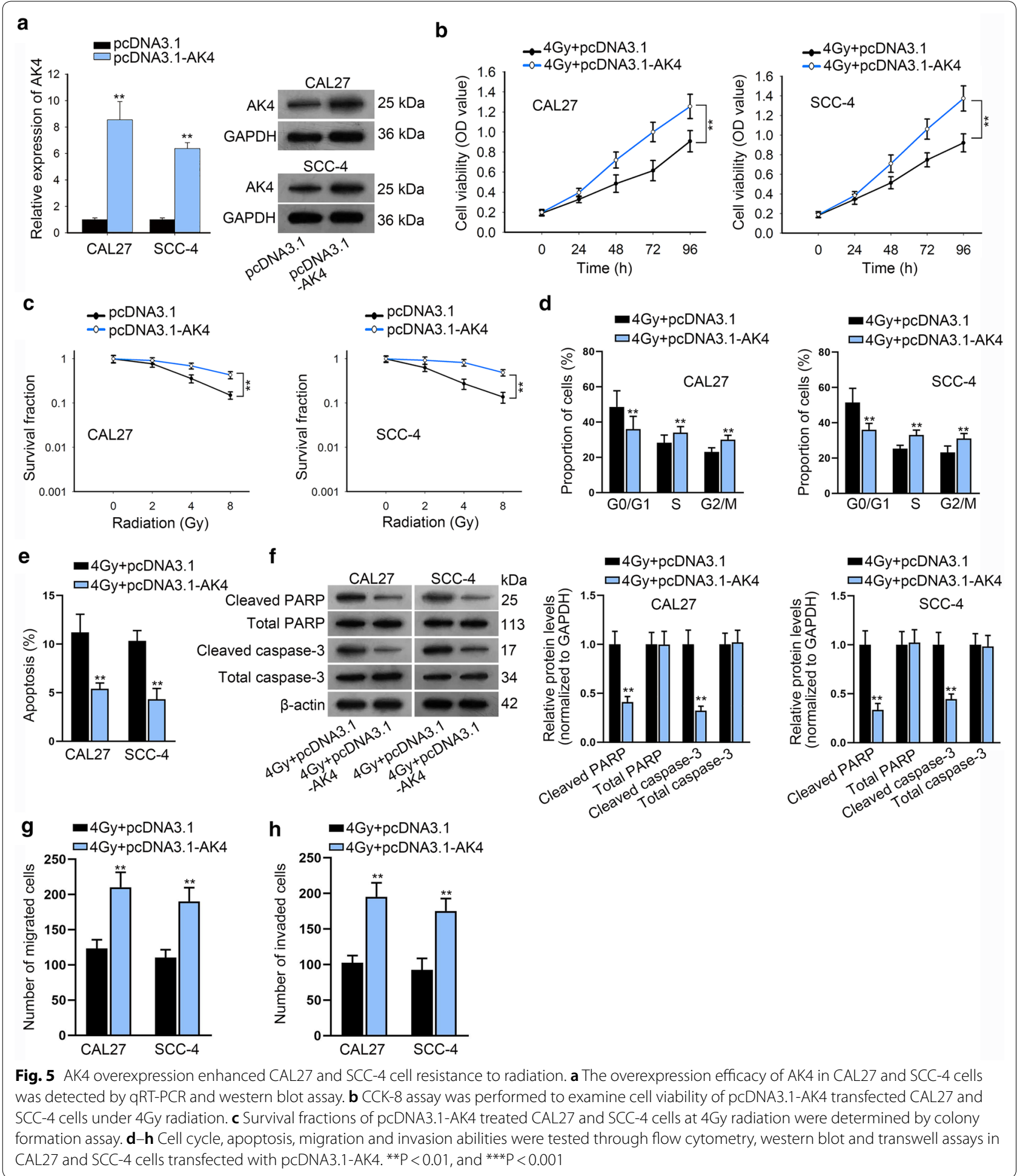

4Gy radiation, cell cycle was arrested when LINC00662 was knocked down whereas, this effect was neutralized when AK4 was overexpressed (Additional file 4: Figure S3A). Flow cytometry analysis illustrated that under $4 \mathrm{~Gy}$ radiation, LINC00662 down-regulation promoted the apoptosis capacity of CAL27 and SCC-4 cells, and this effect was counteracted by AK4 up-regulation (Fig. 6c). Finally, western blot disclosed that after 4Gy radiation, 


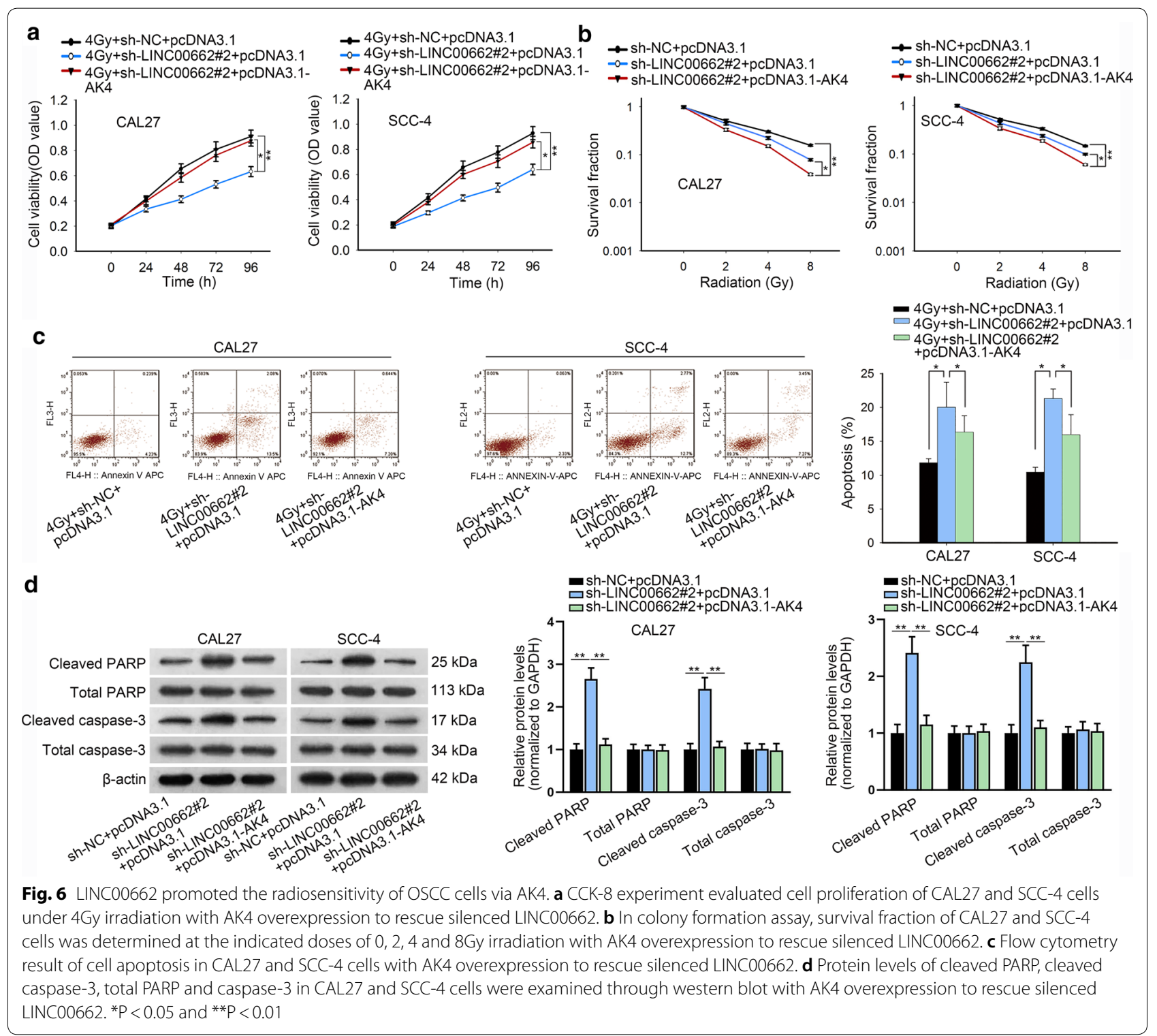

cleaved PARP and cleaved caspase-3 levels were also reduced after LINC00662 was knocked down but partly abolished when AK4 was overexpressed (Fig. 6d). As for cell motility, it was observed from transwell experiments that under 4Gy radiation treatment, the migration and invasion abilities were initially hampered through LINC00662 downregulation, which was repressed through AK4 promotion (Additional file 4: Figure S3B, $\mathrm{C})$. Moreover, we repeated the functional rescue assays using down-regulation of AK4 to rescue LINC00662. AK4 expression was significantly down-regulated at both mRNA and protein levels by transfection of sh-AK4 (Additional file 5: Figure S4A and Additional file 1F). CCK-8 and colony formation assay depicted that silenced
AK4 rescued the promoting effects of LINC00662 overexpression on cell viability and proliferation under radiation exposure (Additional file 5: Figure S4B, C). Flow cytometry cell cycle analysis revealed that, under 4Gy irradiation exposure, cell cycle arrest was inhibited by LINC00662 overexpression, and such effect was counteracted by silenced AK4 (Additional file 5: Figure S4D). Flow cytometry apoptosis assay and western analysis of apoptosis-related proteins revealed that under 4Gy irradiation exposure, silenced AK4 restored the suppressive effects of LINC00662 overexpression on cell apoptosis (Additional file 5: Figure S4E-F). Transwell assay demonstrated that silenced AK4 rescued the promoting effects of LINC00662 overexpression on cell migration 


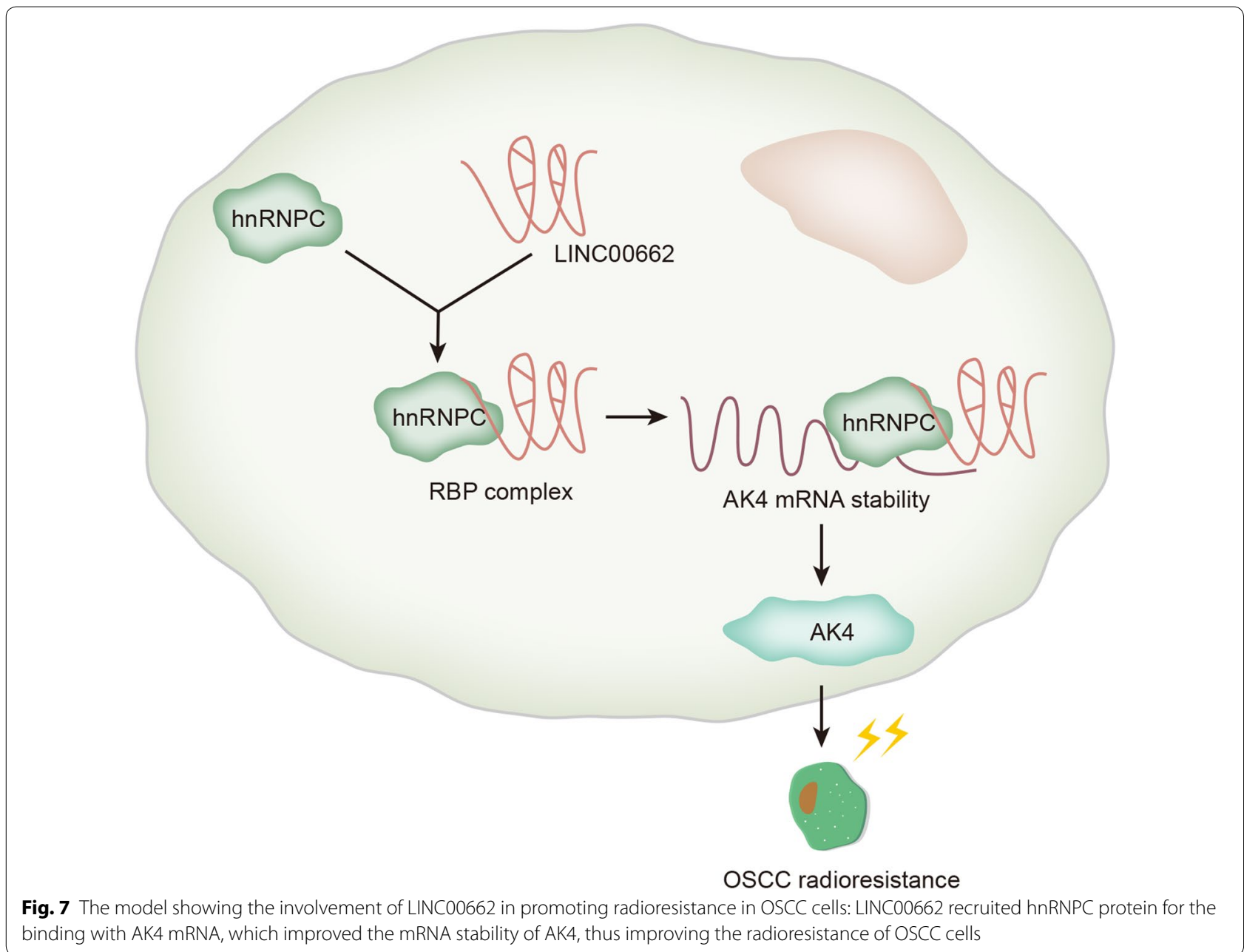

and invasion under 4Gy irradiation exposure (Additional file 5: Figure S4G-H). Overall, LINC00662 served as a scaffold to recruit hnRNPC protein for stabilization of $\mathrm{AK} 4$, and promoted the radiosensitivity of OSCC cells via up-regulation of AK4 (Fig. 7).

\section{Discussion}

Oral squamous cell carcinoma (OSCC) ranks as the eighth commonest oral malignancy in the world [26]. Radiotherapy is one of the main treatments for cancers, however, its therapeutic effect is limited by the radioresistance of tumors, including OSCC [27]. To solve this problem, we need to further investigate the underlying mechanism in OSCC.

Previous studies have demonstrated that lncRNAs are implicated in both physiology and pathological processes of tumors $[28,29]$. The former studies have reported that LINC00662 is a newly identified oncogene and influences cellular processes in OSCC and lung cancer [13, 14]. In the present research, we found the elevated expression of LINC00662 in OSCC cell lines. In addition, the silencing or knockout of LINC00662 could promote the radiosensitivity of OSCC cells by inhibiting cell proliferation, migration and invasion, and inducing apoptosis and cell cycle arrest.

RNA-binding proteins have been unveiled to interact with lncRNAs or target genes, exerting their function in tumors. For example, A FUS (fused in sarcoma)-LATS12 axis restrains hepatocellular carcinoma via activation of Hippo pathway [30]. LncRNA SchLAH inhibits hepatocellular carcinoma metastasis via interacting with FUS [31]. LncRNA H19 boosts tumor growth and predicts a poor prognosis in colorectal cancer through recruiting eIF4A3 [32]. Here, hnRNPC is chosen for investigation. Our paper disclosed the interaction between hnRNPC protein and LINC00662. However, LINC00662 had no regulatory effects on hnRNPC expression. hnRNPC is a survival-related splicing factor in OSCC [18], and served as the tumor facilitator in non-small cell lung cancer [33], ovarian cancer [16]. The hnRNPC protein is one of the 
most abundant pre-mRNA-binding proteins in nucleus and has a single RNP motif RNA-binding domain [34]. hnRNPC serves as the RBP to bind the poly $(\mathrm{U})$ motif and has a genome-wide effect on poly(A) site usage [35]. hnRNPC is known to stabilize uPA mRNA in the nuclear and cytosolic compartments [36]. Besides, hnRNPC was reported to interact with p53 mRNA [37] and protein [17]. Further, AK4, a radioresistant protein in esophageal cancer [20], was revealed in our study to bind with hnRNPC protein and its stability was enhanced by LINC00662. The interplay of hnRNPC protein and AK4 mRNA was firstly affirmed in our study. Overexpression of AK4 was reported to promote lung cancer metastasis [38]. AK4 was involved in hypoxia tolerance and resistance to anti-tumor drugs [39]. AK4 regulates AMPK signaling and its expression is significantly correlated with survival of glioma patients [40]. AK4 promotes lung cancer malignant progression and recurrence at an ATF3-dependent manner [21]. In our study, AK4 promoted OSCC cell proliferation, migration, invasion, and inhibited cell apoptosis and cell cycle arrest; AK4 promoted OSCC cell resistance to irritation exposure.

Moreover, lncRNAs have also been elucidated to influence cellular activities of cancers through interacting with RBPs and further affecting the expression of downstream genes [25]. For instance, lncRNA H19 demonstrates a poor prognosis of colorectal cancer and enhances tumor growth via recruiting eIF4A3 [32]. LINC00324 promotes cell proliferation of gastric cancer cells by binding to $\mathrm{HuR}$ and further stabilizing FAM83B expression [41]. Currently, present study uncovered that LINC00662 promoted AK4 stability by binding to hnRNPC protein, and thus exerted its pro-radioresisitance effects in OSCC cells.

\section{Conclusion}

Finally, rescue experiments suggested that LINC00662 enhanced the radiosensitivity of OSCC cells via hnRNPC-stabilized AK4 through promoting cell proliferation, migration, invasion, and inhibiting cell apoptosis and cell cycle arrest. The current report illustrated the function role and molecular mechanism of LINC00662 in OSCC radiosensitivity, which provided a new direction in researches of tumorigenesis and progression of OSCC.

\section{Supplementary information}

Supplementary information accompanies this paper at https://doi. org/10.1186/s12935-020-01286-9.

Additional file 1 Flow cytometry analysis of transfection efficiency

Additional file 2: Figure S1. LINC00662 modulated the radioresistance of OSCC cells via regulation on cell cycle arrest and cell migration and invasion. (A) Survival fractions of CAL27, SCC-4 cells and NHOK cells at the indicated doses of $0,2,4$ and $8 \mathrm{~Gy}$ radiation were respectively determined by colony formation assay. (B-D) Cell cycle, migration and invasion capabilities were examined via flow cytometry and transwell experiments by LINC00662 silencing. ${ }^{*} P<0.05,{ }^{*} P<0.01$

Additional file 3: Figure S2. LINC00662 knockout reduced radioresistance of OSCC cells. (A) The expression of LINC00662 by transfection of LINC00662-KO in CAL27 and SCC-4 cells was measured by qRT-PCR. (B) CCK-8 assay was performed to examine cell viability of LINC00662-KO transfected CAL27 and SCC-4 cells under 0 or 4Gy radiation, compared with relative control groups. (C) Survival fractions of LINC00662-KO treated CAL27 and SCC-4 cells at the indicated doses of 0, 2, 4 and 8Gy radiation were respectively determined by colony formation assay. (D) Flow cytometry analysis of cell apoptosis in CAL27 and SCC-4 cells with LINC00662 knockout after 0 or 4Gy irradiation treatment. (E) Under 0 or 4Gy irradiation, cleaved PARP, cleaved caspase-3, total PARP and caspase-3 levels in CAL27 and SCC-4 cells with LINC00662 knockout were detected through western blot. $(\mathrm{F}-\mathrm{H})$ Cell cycle, migration and invasion capabilities were examined via flow cytometry and transwell experiments by LINC00662 knockout. ${ }^{* *} \mathrm{P}<0.01$

Additional file 4: Figure S3. Transfection efficiency of plasmids and cell cycle, migration and invasion detection. (A-C) Cell cycle, migration and invasion capabilities were examined via flow cytometry and transwell experiments with AK4 overexpression to rescue silenced LINC00662. ${ }^{*} \mathrm{P}<0.05,{ }^{*} \mathrm{P}<0.01$

Additional file 5: Figure S4. Silenced AK4 rescued the promoting effects of LINC00662 overexpression on the radiosensitivity of OSCC cells. (A) The knockdown efficacy of AK4 in CAL27 and SCC-4 cells was detected by qRT-PCR and western blot assay. (B) CCK-8 experiment evaluated cell proliferation of CAL27 and SCC-4 cells under 4Gy irradiation with AK4 downregulation to rescue LINC00662 overexpression. (C) In colony formation assay, survival fraction of CAL27 and SCC-4 cells was determined at the indicated doses of $0,2,4$ and $8 \mathrm{~Gy}$ irradiation with AK4 down-regulation to rescue LINC00662 overexpression. (D-H) Cell cycle, apoptosis, migration and invasion abilities were tested through flow cytometry, western blot and transwell assays in CAL27 and SCC-4 cells with AK4 down-regulation to rescue LINC00662 overexpression. ${ }^{*} \mathrm{P}<0.05$, ${ }^{*} \mathrm{P}<0.01$

\section{Abbreviations}

OSCC: Oral squamous cell carcinoma; IncRNAs: Long non-coding RNAs; LINC00662: Long intergenic non-protein coding RNA 662; AK4: Adenylate kinase 4; hnRNPC: Heterogeneous nuclear ribonucleoprotein C; qRT-PCR: Quantitative real-time PCR; CCK-8: Cell counting kit-8; RIP: RNA immunoprecipitation; shRNA: Short hairpin RNA; FBS: Fetal bovine serum; SD: Standard deviation; ANOVA: Analysis of variance; PVDF: Polyvinylidene fluoride; SDSPAGE: Sodium dodecyl sulfate-polyacrylamide gel electrophoresis; GAPDH: Glyceraldehyde-3-phosphate dehydrogenase; NC: Negative control.

\section{Acknowledgements}

We thank all participators in this study.

\section{Authors' contributions}

YC: Study design, data curation, article review. CB, XZ, XL and YF: Investigation and preparation, experiment, figures, original article writing. All authors contributed useful advices. All authors read and approved the final manuscript.

\section{Funding}

This work was supported by the Science Technology Department of Zhejiang Province of China (2013C33171) and a program of Wenzhou Science and Technology Bureau (Y20130235).

Availability of data and materials

Research data and material are not shared.

Ethics approval and consent to participate

Not applicable.

Consent for publication

Not applicable. 


\section{Competing interests}

The authors declare that they have no competing interests.

Received: 2 January 2020 Accepted: 22 May 2020

Published online: 16 June 2020

\section{References}

1. Dumache R. Early diagnosis of oral squamous cell carcinoma by salivary microRNAs. Clin Lab. 2017:63:1771-6.

2. Elbers JB, Al-Mamgani A, Paping D, van den Brekel MW, Jóźwiak K, de Boer JP, Karakullukcu B, Verheij M, Zuur CL. Definitive (chemo) radiotherapy is a curative alternative for standard of care in advanced stage squamous cell carcinoma of the oral cavity. Oral Oncol. 2017;75:163-8.

3. Lin C-S, Bamodu OA, Wu A, Chen J-H, Peng Y-J, Cheng M-F, Lee W-H, Hsiao M, Chao T-Y, Yeh C-T. Silencing JARID1B suppresses oncogenicity, stemness and increases radiation sensitivity in human oral carcinoma. Cancer Lett. 2015;368:36-45.

4. Xiao J, Lin L, Luo D, Shi L, Chen W, Fan H, Li Z, Ma X, Ni P, Yang L, et al. Long noncoding RNA TRPM2-AS acts as a microRNA sponge of miR-612 to promote gastric cancer progression and radioresistance. Oncogenesis. 2020;9(3):29.

5. Zhang N, Zeng X, Sun C, Guo H, Wang T, Wei L, Zhang Y, Zhao J, Ma X. LncRNA LINC00963 promotes tumorigenesis and radioresistance in breast cancer by sponging miR-324-3p and inducing ACK1 expression. Mol Therapy Nucleic Acids. 2019;18:871-81.

6. Wang B, Zheng J, Li R, Tian Y, Lin J, Liang Y, Sun Q, Xu A, Zheng R, Liu M, et al. Long noncoding RNA LINC02582 acts downstream of miR-200C to promote radioresistance through CHK1 in breast cancer cells. Cell Death Dis. 2019;10(10):764.

7. Han D, Wang J, Cheng G. LncRNA NEAT1 enhances the radio-resistance of cervical cancer via miR-193b-3p/CCND1 axis. Oncotarget. 2017;9(2):2395-409.

8. Gao C, Lu W, Lou W, Wang L, Xu Q. Long noncoding RNA HOXC13-AS positively affects cell proliferation and invasion in nasopharyngeal carcinoma via modulating miR-383-3p/HMGA2 axis. J Cell Physiol. 2018;234(8):12809-20

9. Hu X, Li Y, Kong D, Hu L, Liu D, Wu J. Long noncoding RNA CASC9 promotes LIN7A expression via miR-758-3p to facilitate the malignancy of ovarian cancer. J Cell Physiol. 2018;234(7):10800-08.

10. Dong Y, Wu W. Downregulation of IncRNA CASC2 promotes the postoperative local recurrence of early oral squamous cell carcinoma. Eur Arch Otorhinolaryngol. 2019;276(2):605-10.

11. Cheng B, Rong A, Zhou Q, Li W. LncRNA LINC00662 promotes colon cancer tumor growth and metastasis by competitively binding with miR-340-5p to regulate CLDN8/IL22 co-expression and activating ERK signaling pathway. J Exp Clin Cancer Res. 2020;39(1):5.

12. Tian $X$, Wu Y, Yang Y, Wang J, Niu M, Gao S, Qin T, Bao D. Long noncoding RNA LINC00662 promotes M2 macrophage polarization and hepatocellular carcinoma progression via activating Wnt/ $\beta$-catenin signaling. Mol Oncol. 2020;14(2):462-83.

13. Sun $\mathrm{P}$, Gong W, Wang $X$, Su Y, Liu Y. Long non-coding RNA Linc00662 promotes cell invasion and contributes to cancer stem cell-like phenotypes in lung cancer cells. J Biochem. 2018;164(6):461-9.

14. Xu D, Chen Y, Yuan C, Zhang S, Peng W. Long non-coding RNA LINC00662 promotes proliferation and migration in oral squamous cell carcinoma. OncoTargets Therapy. 2019;12:647-56.

15. Lixa C, Mujo A, de Magalhães MTQ, Almeida FCL, Lima LMTR, Pinheiro AS. Oligomeric transition and dynamics of RNA binding by the HuR RRM1 domain in solution. J Biomol NMR. 2018;72(3):179-92.

16. Kleemann M, Schneider H, Unger K, Sander P, Schneider EM, FischerPosovszky P, Handrick R, Otte K. MiR-744-5p inducing cell death by directly targeting HNRNPC and NFIX in ovarian cancer cells. Sci Rep. 2018;8(1):9020.

17. Shen Y, Liu S, Fan J, Jin Y, Tian B, Zheng X, Fu H. Nuclear retention of the IncRNA SNHG1 by doxorubicin attenuates hnRNPC-p53 protein interactions. EMBO Rep. 2017;18(4):536-48.

18. Zhang S, Wu X, Diao P, Wang C, Wang D, Li S, Wang Y, Cheng J. Identification of a prognostic alternative splicing signature in oral squamous cell carcinoma. J Cell Physiol. 2020;235(5):4804-13.
19. Lei W, Yan C, Ya J, Yong D, Yujun B, Kai L. MiR-199a-3p affects the multichemoresistance of osteosarcoma through targeting AK4. BMC Cancer. 2018;18(1):631.

20. Zang C, Zhao F, Hua L, Pu Y. The miR-199a-3p regulates the radioresistance of esophageal cancer cells via targeting the AK4 gene. Cancer Cell Int. 2018;18:186.

21. Jan Y-H, Tsai H-Y, Yang C-J, Huang M-S, Yang Y-F, Lai T-C, Lee C-H, Jeng Y-M, Huang C-Y, Su J-L, et al. Adenylate kinase-4 is a marker of poor clinical outcomes that promotes metastasis of lung cancer by downregulating the transcription factor ATF3. Cancer Res. 2012;72(19):5119.

22. Morales J, Li L, Fattah FJ, Dong Y, Bey EA, Patel M, Gao J, Boothman DA. Review of poly (ADP-ribose) polymerase (PARP) mechanisms of action and rationale for targeting in cancer and other diseases. Crit Rev Eukaryot Gene Exp. 2014;24(1):15-28.

23. Nguyen ED, Balas MM, Griffin AM, Roberts JT, Johnson AM. Global profiling of hnRNP A2/B1-RNA binding on chromatin highlights LncRNA interactions. RNA Biol. 2018;15(7):901-13.

24. Zhou X-J, Wu J, Shi L, Li X-X, Zhu L, Sun X, Qian J-Y, Wang Y, Wei J-F, Ding Q. PTEN expression is upregulated by a RNA-binding protein RBM38 via enhancing its mRNA stability in breast cancer. J Exp Clin Cancer Res. 2017;36(1):149

25. Li L, Yin J-Y, He F-Z, Huang M-S, Zhu T, Gao Y-F, Chen Y-X, Zhou D-B, Chen X, Sun L-Q, et al. Long noncoding RNA SFTA1P promoted apoptosis and increased cisplatin chemosensitivity via regulating the hnRNP-U-GADD45A axis in lung squamous cell carcinoma. Oncotarget. 2017:8(57):97476-89.

26. Siegel R, Ward E, Brawley O, Jemal A. Cancer statistics, 2011. CA Cancer J Clin. 2011;61(4):212-36.

27. Krüger M, Amort J, Wilgenbus P, Helmstädter JP, Grechowa I, Ebert J, Tenzer S, Moergel M, Witte I, Horke S. The anti-apoptotic PON2 protein is Wnt/ $\beta$-catenin-regulated and correlates with radiotherapy resistance in OSCC patients. Oncotarget. 2016;7(32):51082-95.

28. Fatica A, Bozzoni I. Long non-coding RNAs: new players in cell differentiation and development. Nat Rev Genet. 2013;15:7.

29. Dong J, Teng F, Guo W, Yang J, Ding G, Fu Z. IncRNA SNHG8 promotes the tumorigenesis and metastasis by sponging miR-149-5p and predicts tumor recurrence in hepatocellular carcinoma. Cell Physiol Biochem. 2018:51(5):2262-74

30. Bao L, Yuan L, Li P, Bu Q, Guo A, Zhang H, Cui N, Liu B. A FUS-LATS1/2 axis inhibits hepatocellular carcinoma progression via activating hippo pathway. Cell Physiol Biochem. 2018;50(2):437-51.

31. Ge Z, Cheng Z, Yang X, Huo X, Wang N, Wang H, Wang C, Gu D, Zhao F, Yao M, et al. Long noncoding RNA SchLAH suppresses metastasis of hepatocellular carcinoma through interacting with fused in sarcoma. Cancer Sci. 2017;108(4):653-62.

32. Han D, Gao X, Wang M, Qiao Y, Xu Y, Yang J, Dong N, He J, Sun Q, Lv G, et al. Long noncoding RNA H19 indicates a poor prognosis of colorectal cancer and promotes tumor growth by recruiting and binding to elF4A3. Oncotarget. 2016;7(16):22159-73.

33. Yan M, Sun L, Li J, Yu H, Lin H, Yu T, Zhao F, Zhu M, Liu L, Geng Q, et al. RNA-binding protein KHSRP promotes tumor growth and metastasis in non-small cell lung cancer. J Exp Clin Cancer Res. 2019;38(1):478.

34. Wan L, Kim JK, Pollard VW, Dreyfuss G. Mutational definition of RNAbinding and protein-protein interaction domains of heterogeneous nuclear RNP C1. J Biol Chem. 2001;276(10):7681-8.

35. Gruber AJ, Schmidt R, Gruber AR, Martin G, Ghosh S, Belmadani M, Keller W, Zavolan M. A comprehensive analysis of $3^{\prime}$ end sequencing data sets reveals novel polyadenylation signals and the repressive role of heterogeneous ribonucleoprotein C on cleavage and polyadenylation. Genome Res. 2016;26(8):1145-59.

36. Shetty S. Protein synthesis and urokinase mRNA metabolism. Mol Cell Biochem. 2005;271:13-22

37. Christian KJ, Lang MA, Raffalli-Mathieu F. Interaction of heterogeneous nuclear ribonucleoprotein $\mathrm{C} 1 / \mathrm{C} 2$ with a novel cis-regulatory element within p53 mRNA as a response to cytostatic drug treatment. Mol Pharmacol. 2008:73(5):1558-67.

38. Jan YH, Lai TC, Yang CJ, Lin YF, Hsiao MS, Hsiao M. Adenylate kinase 4 modulates oxidative stress and stabilizes HIF-1 a to drive lung adenocarcinoma metastasis. J Hematol Oncol. 2019;12(1):12.

39. Fujisawa K, Terai S, Takami T, Yamamoto N, Yamasaki T, Matsumoto T, Yamaguchi K, Owada Y, Nishina H, Noma T, et al. Modulation of 
anti-cancer drug sensitivity through the regulation of mitochondrial activity by adenylate kinase 4. J Exp Clin Cancer Res. 2016;35:48.

40. Lanning NJ, Looyenga BD, Kauffman AL, Niemi NM, Sudderth J, DeBerardinis RJ, MacKeigan JP. A mitochondrial RNAi screen defines cellular bioenergetic determinants and identifies an adenylate kinase as a key regulator of ATP levels. Cell Rep. 2014;7(3):907-17.

41. Zou Z, Ma T, He X, Zhou J, Ma H, Xie M, Liu Y, Lu D, Di S, Zhang Z Long intergenic non-coding RNA 00324 promotes gastric cancer cell proliferation via binding with HuR and stabilizing FAM83B expression. Cell Death Dis. 2018;9(7):717.

\section{Publisher's Note}

Springer Nature remains neutral with regard to jurisdictional claims in published maps and institutional affiliations.
Ready to submit your research? Choose BMC and benefit from:

- fast, convenient online submission

- thorough peer review by experienced researchers in your field

- rapid publication on acceptance

- support for research data, including large and complex data types

- gold Open Access which fosters wider collaboration and increased citations

- maximum visibility for your research: over $100 \mathrm{M}$ website views per year

At BMC, research is always in progress.

Learn more biomedcentral.com/submissions 